International Journal of Biomedicine I June 2019 - Volume 9, Issue Suppl_1: Abstracts From the Second Russian International Conference "Cryo-electron microscopy 2019: achievements and prospects"

\begin{abstract}
ORAL ABSTRACT PRESENTATIONS
SESSION TITLE: STRUCTURE AND FUNCTIONS OF THE TRANSCRIPTION AND TRANSLATION APPARATUS OF THE CELL
\end{abstract}

DOI: 10.21103/IJBM.9.Suppl_1.OR3

\title{
Abstract OR-3: Fleeting Ensembles: Transient Protein-Protein Interactions in DNA Repair
}

\author{
Dmitry O. Zharkov \\ Novosibirsk State University, Novosibirsk, Russia
}

Background: DNA repair is a vitally important process that protects the cell's genome from ongoing damage. Six DNA repair pathways are commonly outlined: direct reversal, base excision repair, nucleotide excision repair, mismatch repair, recombination repair, and non-homologous end joining. Of these, base excision repair that deals with small non-bulky lesions removes the greatest number of damaged nucleotides daily. Unlike many other cellular pathways, base excision repair is not carried out by multienzyme complexes but rather involves many DNA-mediated transient protein-protein interactions that pass the damaged DNA between consecutive participants of the pathway.

Methods: Direct structural characterization of such DNA substrate channeling remains elusive, and most of what is known about it was obtained through enzyme kinetics and modeling.

Results: DNA glycosylases, the enzymes that initiate base excision repair, are actively displaced from their enzyme-product complexes by abasic site (AP) endonucleases, the next enzyme in the pathway. Several accessory proteins, such as XRCC1, PCNA, and PARP1, guide the dynamic assembly and disassembly of DNA repair complexes providing regulatory modifications and scaffolding. Interestingly, eukaryotic and bacterial DNA repair proteins are often homologous but the eukaryotic proteins carry additional extended intrinsically disordered domains, which are believed either to be involved into dynamic protein-protein interactions or provide additional DNA-binding capabilities outside the welldefined DNA binding domains.

Conclusion: Overall, DNA repair, and especially base excision DNA repair, relies on extensive conformational rearrangements of the participating proteins, which is necessary both for damage recognition and for progress along the repair pathway. As such, they represent a promising target for studies with cryo-EM techniques.

Key words: DNA damage $\bullet$ DNA repair $\bullet$ conformational ensembles $\bullet$ protein-protein interactions

Sources of Funding: This work was supported by the Russian State Foundation (Grant 17-14-01190)

International Journal of Biomedicine. 2019;9 Suppl 1: S6-7. doi: 10.21103/IJBM.9.Suppl_1.OR3

C2019 International Medical Research and Development Corporation 